\title{
植物反射光谱对水分生理变化响应的研究进展
}

\author{
刘 畅 孙鹏森* 刘世荣
}

中国林业科学研究院森林生态环境与保护研究所, 国家林业局森林生态环境重点实验室, 北京 100091

\begin{abstract}
摘 要 实时、无损伤地探测植物的水分及生理变化是高光谱遥感的深层次应用。由水分胁迫引发的植物一系列反射光谱响 应体现了碳-氮-水塊合作用的结果。以往的研究大多集中于单一因素的响应, 而忽略了多因素交互作用。该文综述和分析了 植物水分状况变化引起的直接和间接光谱响应机制, 包括植物水分含量、色素、养分状况、光合作用和叶绿素荧光指标的光 谱响应及其内在的关联, 探讨了反射光谱在探测植物水分生理活动应用中的主要方法与最新技术, 并指出碳-氮-水多指标、 多时空尺度的综合分析对于估测植被生产力及其对气候变化的响应具有重要意义。
\end{abstract}

关键词 植物反射光谱; 水分胁迫; 水分生理; 色素; 营养元素; 光合作用; 叶绿素荧光

引用格式: 刘畅, 孙鹏森, 刘世荣 (2016). 植物反射光谱对水分生理变化响应的研究进展. 植物生态学报, 40, 80-91. doi: 10.17521/cjpe.2015.0267

\section{A review of plant spectral reflectance response to water physiological changes}

LIU Chang, SUN Peng-Sen*, and LIU Shi-Rong

Institute of Forest Ecology, Environment and Protection, Chinese Academy of Forestry, Key Laboratory of Forest Ecology and Environment, State Forestry Administration, Beijing 100091, China

\begin{abstract}
Spectral reflectance is a new, real time and non-destructive hyperspectral remote sensing application to monitor plant water status and physiological changes. The spectral reflectance responses induced by water stress reflect the interaction and coupling of carbon, nitrogen and water cycles. A majority of previous studies focused on a specific structural or physiological effect on spectral reflectance with little attention on their interactions. This paper reviewed and synthesized the direct and indirect spectral responses caused by changes in plant water content, pigments, nutrient status, photosynthesis and chlorophyll fluorescence indices and their internal association. This paper also discussed the common approaches and the new techniques in applying spectral reflectance for detecting water status and physiological activities in plants. This paper concluded that analysis of the spectral reflectance at multiple temporal or spatial scales might have a potential application in projecting vegetation productivities, particularly in the context of climate change.
\end{abstract}

Key words plant spectral reflectance; water stress; water physiology; pigment; nutrient element; photosynthesis; chlorophyll fluorescence

Citation: Liu C, Sun PS, Liu SR (2016). A review of plant spectral reflectance response to water physiological changes. Chinese Journal of Plant Ecology, 40, 80-91. doi: 10.17521/cjpe.2015.0267

遥感是一种非损伤性地获得被测物体信息的手 段, 20 世纪 80 年代兴起的高光谱遥感在生态学上的 应用扩展了我们认识植被的能力, 使我们能够由原 来的简单辨识植被 “是什么”逐步发展到定量化估测 植被“怎么样”的更高水平(Muttiah, 2002)。早期我们 更多地利用遥感手段获取叶面积指数 $(L A I)$ 、归一化 植被指数 $(N D V I)$ 等植被的分类或结构信息, 而现在 越来越多的科学家开始关注遥感在估算植被生理参 数方面的潜力 (Zarco-Tejada et al., 2003a; Dobrowski et al., 2005)。正如Grace等(2007)所言: “这是一项富 有挑战且激动人心的工作”, 因为这对于陆地生态 系统光合生产力、水分循环(Sellers et al., 1997; Szilagyi, 2000)和碳循环(Veroustraete et al., 2002)等 研究领域非常重要, 实际上, 美国从2003年已经开 始倡导建立一种所谓的光谱网络(SpecNet), 和现有 的通量网络水碳观测同步进行(Gamon et al., 2006)。 目前国际上对植被反射光谱和生理参数之间的定量 关系已经有了一定的认识, 但在不同尺度上这些定

收稿日期Received: 2015-07-13 接受日期Accepted: 2015-10-23

* 通信作者Author for correspondence (E-mail: sunpsen@caf.ac.cn) 
量关系的稳定性还有待验证, 仍需要从试验室的受 控条件下植物生理变化的反射光谱响应研究开始 (Ustin et al., 1998; Li et al., 2000), 将试验室开发的 反射光谱指数和卫星遥感相结合, 逐步测试并应用 到宏观尺度, 这在技术上被证明是可行的。在叶片 尺度上, Jacquemoud和Biswal (1990)基于Allen平板 模型发展起来的PROSPECT模型是应用最多的叶片 辐射传输模型。该模型需要输入两类参数: 叶片结 构参数和叶片的生化参数含量, 包括色素含量、等 效水分厚度 $(E W T)$ 、干物质含量等, 模型前向运算可 以得到叶片 400-2 $500 \mathrm{~nm}$ 的半球反射率和透射率, 反演则可以估算叶片的生化参数。在冠层尺度上, Verhoef (1984)在改进Suits 模型的基础上提出的 SAIL模型是最早的一个冠层模型, 该模型所需要输 入的参数包括叶片反射率和透射率、冠层结构参数 和观测几何数据。Baret等(1992)首次将SAIL模型与 PROSPECT模型结合, 提出PROSAIL模型, 这使得 在不同尺度上反演植物的生理生化参数成为可能。 而在像元尺度上, Zarco-Tejada等(2003b)利用叶片冠层的辐射传输模型模拟的等效MODIS反射率光 谱分析冠层结构、观测几何等在估测叶片含水量中 的影响, 最后利用同步的MODIS数据反演得到叶片 含水量, 其反演结果和实测值具有良好的相关性。 当前, 由水分胁迫引发的叶绿素荧光、碳同化、色 素转化等生理变化的反射光谱响应具有一定的复杂 性和内在耦联性, 需要我们综合目前分散的研究, 进行深入和系统的分析。

\section{1 植物水分状况及对反射光谱的影响规律}

水分是植物叶片的重要组成部分, 占叶片鲜质 量的50\%-80\%, 水分自身的光谱吸收范围覆盖近红 外和短波红外区间(NIR \& SWIR), 比较显著的吸收 峰(谷)有970、1 200、1450、1 950和2 $250 \mathrm{~nm}$ (Hunt \& Rock, 1989; Rollin \& Milton, 1998), 因此, 叶片含 水量的变化对植物NIR 和SWIR区域的光谱反射率 的影响很大(Hunt \& Rock, 1989; Yu et al., 2000; Ceccato et al., 2001; Imanishi et al., 2004)。通常, 引 起植物叶片反射光谱发生变化的因素分为结构性因 素(叶片厚度、叶片干物质、水分含量等)和生理性 因素(色素浓度、叶氮含量、光合速率、气孔行为和 叶绿素苂光等)(Muttiah, 2002)。水分一方面被看作 是一个影响反射光谱的结构性因素, 是植物叶片的
重要组成部分, 在NIR和SWIR反射区具有特定的光 谱吸收区间; 另一方面, 水分的变化又诱导植物叶 片的生理变化, 引起反射光谱中可见光区(VIS)的变 化, 这充分体现了水分胁迫对光谱影响的复杂性。

植物水分胁迫的光谱响应研究最早出现在测谱 学领域, 主要目的是构建植物水分敏感性光谱指数, 迄今已经开发出许多光谱指数用以估测植物水分含 量的变化(表1)。

在叶片尺度上, Bowman (1989)在对棉花 (Gossypium spp.)叶片的水分状况和光谱反射率的研 究中发现近红外区反射光谱随着含水量的降低而升 高, 其中810、1665和2 $210 \mathrm{~nm}$ 处与叶片的相对含水 量 $(R W C)$ 、叶片总水势 $\left(\Psi_{\mathrm{PD}}\right)$ 和膨压具有显著的相关 关系。Carter (1993)系统地研究了6种不同种类植物 叶片在不同的水分含量下对叶片光谱反射特性的影 响, 发现水分含量对1 300-2 500 nm波段反射率的 影响是由水分对辐射的直接吸收造成的, 对 400-1 $300 \mathrm{~nm}$ 波段反射率的影响则是由水分含量造 成的叶片内部结构的变化引起的。除了对叶片的反 射光谱本身与水分含量的关系的分析研究, 还有学 者考虑了反射光谱曲线的导数在其中的应用, Danson等(1992)的研究发现叶片在1 360-1 $470 \mathrm{~nm}$ 和 1830-2 080 nm等波段处反射率的一阶导数与叶片 含水量高度相关, 而且对叶片结构的影响不敏感。 $\mathrm{Yu}$ 等(2000)利用叶片失水试验, 用叶片 $R W C$ 、比叶 含水量 $(S W C)$ 、单位叶片含水量 $(L W C)$ 等指标表征草 本植物和木本树种叶片的水分状态, 进而建立了叶 片反射光谱和水分状态之间的关系。沈艳等(2005) 通过测定单子叶和双子叶植物的叶片含水量和高光 谱反射率, 分别建立两种植物叶片含水量的单变量 和多变量估算模型, 发现总体上单子叶植物的反演 精度高于双子叶植物。为了找出能把水分胁迫与其 他生物胁迫区分开来的光谱波段或光谱组合, Graeff和Claupein (2007)对不同水分处理下的小麦 (Triticum aestivum) 叶片光谱数据进行了研究分析, 发现无论在哪个生长阶段反射模式在 $510_{780} \mathrm{~nm}$ (指 的是波长范围为 $510-780 \mathrm{~nm}$ 的光谱反射率)、 $540_{780}$ $\mathrm{nm} 、 490_{1300} \mathrm{~nm}$ 和 $540_{1300} \mathrm{~nm}$ 的波段最适合来表示小 麦的叶片水分状况。Osório等(2012)对不同温度和水 分组合条件下长角果(Ceratonia siliqua)幼苗的研究 表明: 与叶片 $\Psi_{\mathrm{PD}}$ 和 $R W C$ 相比, 长角果叶片的水浓 度 $(W C)$ 和水分指数 $(W I)$ 的相关性更高。潘佩芬等 
表1 植物生理指标相关的光谱参数及其定义

Table 1 Spectral parameters and their definitions related to physiological indices of plant

\begin{tabular}{|c|c|c|c|c|}
\hline $\begin{array}{l}\text { 类型 } \\
\text { Type }\end{array}$ & $\begin{array}{l}\text { 光谱参数 } \\
\text { Spectral parameter }\end{array}$ & $\begin{array}{l}\text { 缩写 } \\
\text { Acronym }\end{array}$ & $\begin{array}{l}\text { 定义 } \\
\text { Definition }\end{array}$ & $\begin{array}{l}\text { 参考文献 } \\
\text { Reference }\end{array}$ \\
\hline \multirow[t]{6}{*}{$\begin{array}{l}\text { 水分相关 } \\
\text { Related to water }\end{array}$} & \multirow[t]{2}{*}{$\begin{array}{l}\text { 水分指数 } \\
\text { Water index }\end{array}$} & WI & $R_{970} / R_{900}$ & $\begin{array}{l}\text { Peñuelas et al., } \\
\text { 1997b }\end{array}$ \\
\hline & & $W I / N D V I$ & $\left(R_{970} / R_{900}\right) /\left[\left(R_{900}-R_{680}\right) /\left(R_{900}+R_{680}\right)\right]$ & $\begin{array}{l}\text { Peñuelas \& Inoue, } \\
1999\end{array}$ \\
\hline & 冠层结构指数 & \multirow[t]{2}{*}{ CSI } & $2 s S R-s S R^{2}+s W I^{2}$ & \multirow{2}{*}{$\begin{array}{l}\text { Sims \& Gamon, } \\
2003\end{array}$} \\
\hline & Canopy structure index & & $\begin{array}{l}\text { where: } s S R=\left(R_{800} / R_{680}-1\right) /\left(R_{800} / R_{680}-1\right)_{\max } \\
s W I=\left(R_{900} / R_{1180}-1\right) /\left(R_{900} / R_{1180}-1\right)_{\max }\end{array}$ & \\
\hline & 归一化水分指数 & $N D W I$ & $\left(R_{860}-R_{1240}\right) /\left(R_{860}+R_{1240}\right)$ & Gao, 1996 \\
\hline & & $\begin{array}{l}S R_{(610,560)} \\
N D_{(810,610)}\end{array}$ & $\left(R_{610} / R_{560}\right) /\left[\left(R_{810}-R_{610}\right) /\left(R_{810}+R_{610}\right)\right]$ & Tian et al., 2004 \\
\hline \multirow{21}{*}{$\begin{array}{l}\text { 色素相关 } \\
\text { Related to } \\
\text { pigment }\end{array}$} & $\begin{array}{l}\text { 归一化植被指数 } \\
\text { Normalized difference vegetation index }\end{array}$ & $N D V I$ & $\left(R_{800}-R_{680}\right) /\left(R_{800}+R_{680}\right)$ & Rouse et al., 1974 \\
\hline & $\begin{array}{l}\text { 绿色归一化植被指数 } \\
\text { Green normalized difference vegetation index }\end{array}$ & $\begin{array}{l}\text { Green } \\
N D V I\end{array}$ & $\left(R_{750}-R_{550}\right) /\left(R_{750}+R_{550}\right)$ & $\begin{array}{l}\text { Gitelson \& } \\
\text { Merzlyak, } 1994\end{array}$ \\
\hline & $\begin{array}{l}\text { 改良红边比值指数 } \\
\text { Modified red-edge simple ratio index }\end{array}$ & $m S R_{705}$ & $\left(R_{750}-R_{445}\right) /\left(R_{705}-R_{445}\right)$ & $\begin{array}{l}\text { Sims \& Gamon, } \\
2002\end{array}$ \\
\hline & $\begin{array}{l}\text { 改良红边归一化植被指数 } \\
\text { Modified red-edge normalized difference vegetation index }\end{array}$ & $m N D_{705}$ & $\left(R_{750}-R_{705}\right) /\left(R_{750}+R_{705}-2 R_{445}\right)$ & $\begin{array}{l}\text { Sims \& Gamon, } \\
2002\end{array}$ \\
\hline & \multirow{3}{*}{$\begin{array}{l}\text { 色素简单比值指数 } \\
\text { Pigment specific simple ratio }\end{array}$} & PSSRa & $R_{800} / R_{680}$ & \multirow[t]{3}{*}{ Blackburn, 1998} \\
\hline & & PSSRb & $R_{800} / R_{635}$ & \\
\hline & & PSSRc & $R_{800} / R_{470}$ & \\
\hline & \multirow{3}{*}{$\begin{array}{l}\text { 色素归一化指数 } \\
\text { Pigment specific normalized difference }\end{array}$} & $P S N D a$ & $\left(R_{800}-R_{680}\right) /\left(R_{800}+R_{680}\right)$ & \multirow[t]{3}{*}{ Blackburn, 1998} \\
\hline & & $P S N D b$ & $\left(R_{800}-R_{635}\right) /\left(R_{800}+R_{635}\right)$ & \\
\hline & & $P S N D c$ & $\left(R_{800}-R_{470}\right) /\left(R_{800}+R_{470}\right)$ & \\
\hline & \multirow{3}{*}{$\begin{array}{l}\text { 反射光谱比值分析 } \\
\text { Ratio analysis of reflectance spectra }\end{array}$} & $R A R S a$ & $\left(R_{650} / R_{700}\right) /\left(r_{650} / r_{700}\right)$ & \multirow{3}{*}{$\begin{array}{l}\text { Chappelle et al., } \\
1992\end{array}$} \\
\hline & & $R A R S b$ & $\left(R_{700} \times R_{675} / R_{650}\right) \times\left(r_{700} \times r_{675} / r_{650}\right)$ & \\
\hline & & $R A R S c$ & $\left(R_{760} / R_{500}\right) /\left(r_{760} / r_{500}\right)$ & \\
\hline & \multirow{4}{*}{$\begin{array}{l}\text { 结构不敏感色素指数 } \\
\text { Structure-insensitive pigment index } \\
\text { 植物衰老反射指数 } \\
\text { Plant senescence reflectance index } \\
\text { 类胡萝卜素反射指数 } \\
\text { Carotenoid reflectance indices }\end{array}$} & SIPI & $\left(R_{800}-R_{445}\right) /\left(R_{800}-R_{680}\right)$ & \multirow{4}{*}{$\begin{array}{l}\text { Peñuelas et al., } \\
\text { 1995a } \\
\text { Merzlyak et al., } \\
1999 \\
\text { Gitelson et al., } \\
2002\end{array}$} \\
\hline & & PSRI & $\left(R_{678}-R_{550}\right) / R_{750}$ & \\
\hline & & $C R I_{550}$ & $1 / R_{510}-1 / R_{550}$ & \\
\hline & & $C R I_{700}$ & $1 / R_{510}-1 / R_{700}$ & \\
\hline & $\begin{array}{l}\text { 改良的类胡萝卜素反射指数 } \\
\text { Modified carotenoid reflectance index }\end{array}$ & $m C R I$ & $R_{780} /\left[\left(1 / R_{510}\right)-\left(1 / R_{550}\right)\right]$ & $\begin{array}{l}\text { Gitelson et al., } \\
2006\end{array}$ \\
\hline & $\begin{array}{l}\text { 归一化光谱反射指数 } \\
\text { Normalized difference spectral reflectance index }\end{array}$ & $N D_{(1536,707)}$ & $\left(R_{1536}-R_{707}\right) /\left(R_{1536}+R_{707}\right)$ & Wang et al., 2009 \\
\hline & $\begin{array}{l}\text { 光谱反射指数 } \\
\text { Spectral reflectance index }\end{array}$ & $S R_{(723,770)}$ & $R_{723} / R_{770}$ & Yang et al., 2010 \\
\hline & $\begin{array}{l}\text { 归一化光谱反射指数 } \\
\text { Normalized difference spectral reflectance index }\end{array}$ & $N D_{(770,713)}$ & $\left(R_{770}-R_{713}\right) /\left(R_{770}+R_{713}\right)$ & \\
\hline \multirow{4}{*}{$\begin{array}{l}\text { 营养元素相关 } \\
\text { Related to nu- } \\
\text { trient element }\end{array}$} & 植株氮光谱指数 Plant nitrogen spectral index & PNSI & $|(N I R+r e d) /(N I R-r e d)|$ & Stone et al., 1996 \\
\hline & \multirow{2}{*}{$\begin{array}{l}\text { 比值植被指数 } \\
\text { Ratio vegetation indices }\end{array}$} & $R V I_{(870,660)}$ & $R_{870} / R_{660}$ & \multirow[t]{2}{*}{ Zhu et al., 2008} \\
\hline & & $R V I_{(810,660)}$ & $R_{810} / R_{660}$ & \\
\hline & $\begin{array}{l}\text { 归一化光谱反射指数 } \\
\text { Normalized difference spectral reflectance index }\end{array}$ & $N D_{(483,503)}$ & $\left(R_{483}-R_{503}\right) /\left(R_{483}+R_{503}\right)$ & $\begin{array}{l}\text { Stroppiana et al., } \\
2009\end{array}$ \\
\hline \multirow{4}{*}{$\begin{array}{l}\text { 光合和叶绿素 } \\
\text { 若光相关 } \\
\text { Related to pho- } \\
\text { tosynthesis and } \\
\text { chlorophyll } \\
\text { fluorescence }\end{array}$} & 生理反射指数 & \multirow[t]{2}{*}{ PRI } & $\left(R_{531}-R_{570}\right) /\left(R_{531}+R_{570}\right)$ & \multirow[t]{2}{*}{ Gamon et al., 1992} \\
\hline & Physiological reflectance index & & & \\
\hline & \multirow{2}{*}{$\begin{array}{l}\text { 荧光比值指数 } \\
\text { Fluorescence ratio indices }\end{array}$} & \multirow[t]{2}{*}{ FRI } & $R_{600} / R_{690}$ & \multirow{2}{*}{$\begin{array}{l}\text { Dobrowski et al., } \\
2005\end{array}$} \\
\hline & & & $R_{740} / R_{800}$ & \\
\hline
\end{tabular}

NIR, 近红外光区的; red, 红光区间; $R_{\lambda}, \lambda \mathrm{nm}$ 的光谱反射率; $r_{\lambda}, \lambda \mathrm{nm}$ 的光谱反射率的平均值。

$N I R$, near infrared region; red, red light region; $R_{\lambda}$, spectral reflectance of $\lambda \mathrm{nm} ; r_{\lambda}$, mean of spectral reflectance of $\lambda \mathrm{nm}$. 
(2013)运用主成分分析的方法研究棕榈(Trachycarpus fortunei) 叶片的反射光谱与含水率(叶片含水 率 $=$ (叶片鲜质量 - 叶片干质量 $) /$ 叶片鲜质量 $\times 100 \%$ ) 的关系, 发现454、668、1 466、1 664和1 $924 \mathrm{~nm}$ 波 段处与含水率显著相关, 并建立了精度较高的植被 含水率模型。此外, 还有一些学者建立了基于叶片 干质量的水分指标(Gao \& Goetz, 1995; Ustin et al., 1998)和 EWT指标(Ceccato et al., 2001)等, 进一步揭 示了水分和光谱反射率之间的关系。但是, 从植物 生理学早期的定义来看, $R W C$ 指标具有较强的生理 学意义, 同时大量的研究证实 $R W C$ 与植物的蒸腾和 气孔行为等生理活动之间的相关性高于其他含水量 指标(Tyree \& Jarvis, 1982; Kramer \& Boyer, 1995), $R W C$ 在探索水分变化引起植物生理变化、进而对反 射光谱造成影响的研究方面具有一定的优势。

在冠层尺度上, Holben等(1983)发现干旱时大 豆(Glycine max) 的冠层光谱反射率在630-690 nm、 760-900 nm和1 550-1 $750 \mathrm{~nm}$ 波段变化明显, 并指 出760-900 nm波段为探测水分胁迫的最佳波段。 Inoue等(1993)研究认为950-970 $\mathrm{nm}$ 处水分弱吸收谷 的反射率随含水量的变化在叶片和冠层水平都比较 明显, 进而提出了用950-970 nm处的反射率来预估 冠层叶片的相对含水量。Peñuelas等(1997b)发现用 $W I$ 、近红外波段或其一阶导数的最小值等红边参数 能清楚地指示水分状况的变化。随后, Peñuelas和 Inoue (1999)的研究表明, WI与 $N D V I$ 的比值在预测 叶片水分含量的基础上, 还可以用来预测植株或冠 层的含水量或水势。Sims和Gamon (2003)从光谱反 射率的角度综合了 $W I$ 等指数对浓密与稀疏冠层探 测能力的差异, 提出冠层结构指数 $(C S I)$ 反演植被含 水量。Gao (1996)在研究中发现 $1240 \mathrm{~nm}$ 处的光谱特 征峰对水分含量变化非常敏感, 而860 nm处则非常 不敏感, 故由此提出归一化水分指数 $(N D W I)$ 。在时 间尺度上, 田永超等(2004)研究发现, 在小麦整个 生长期内, 其冠层叶片含水率与460-1 $500 \mathrm{~nm}$ 波段 范围内的光谱反射率均有良好的相关性, 植株(冠 层叶片和茎鞘)含水率与560-1480 nm波段范围内 光谱反射率的相关性均达到极显著水平, 其中, 用 光谱指数 $\left(S R_{(610,560)} / N D_{(810,610)}\right)$ 监测不同生育期小麦 冠层叶片和植株含水率的效果最好。

此外, 光谱遥感也广泛用于大尺度的作物干旱 监测。Sandholt等(2002)利用地表温度 $\left(T_{\mathrm{S}}\right)$ 和 $N D V I$ 构
建了温度植被干旱指数 $(T V D I)$ 用以监测植被的干旱 状况, 结果表明TVDI的变化比水文模型能更好地反 映水分和干旱的变化。张洁等(2012)在华北平原中 部地区的研究表明植被供水指数 $(V S W I)$ 在整个冬小 麦生长季时间序列上干旱监测的稳定性最好, 并指 出当研究区域扩大到包括多种作物时就必须考虑作 物间差异对监测结果的影响。王治海等(2013)将遥 感信息与作物干旱模型相结合, 提高了原有作物干 旱模型对冬小麦生长发育的模拟能力, 并且模型对 区域作物干旱的模拟结果也更加接近实际情况。徐 焕颖等(2015)基于中分辨率成像光谱仪(MODIS)数 据, 结合温度和降水数据, 采用改进归一化水分指 数 $(M N D W I$ 等对黄淮海平原2001-2012年干早情况 进行监测, 并分析了其空间、季节、年际变化规律 及其原因。

\section{2 植物色素与反射光谱的关系}

光合作用过程中吸收光能的植物色素包括叶绿 素和类胡萝卜素, 前者是主要的吸收光能物质, 直 接影响植物光合作用的光能利用, 后者有保护叶绿 素的功能, 因此色素是植物与外界进行物质能量交 换的物质基础。叶绿素含量和植被的光合能力、发 育阶段有较好的相关性, 它们往往是光合作用能力 和植被发育阶段 (特别是衰老阶段) 的指示器 (Minolta, 1989)。水分胁迫可导致叶绿素含量降低, 随着胁迫强度的增加和时间的延长, 叶绿素降解加 剧, 降解原因主要是活性氧、过氧化氢及丙二醛含 量增加, 抗氧化剂如抗坏血酸、还原型谷胱甘肽及 胡萝卜素含量下降, 从而导致叶绿素蛋白复合体功 能受到损伤。

\section{1 叶绿素}

叶绿素(Chl)作为光合作用过程中最主要的参 与者, 直接控制着植物能量和物质的循环。利用高 光谱遥感技术测得的植物反射光谱来检测植被 Chl 含量的变化已经有较多的研究。Rouse等(1974)研究 了植被反射光谱与 $\mathrm{Chl}$ 含量的关系, 证明 $N D V I$ 与 $\mathrm{Chl}$ 的含量成正比。但在Chl含量较高的植物叶片中, 色 素变化的敏感性在675 nm波段处最低, 由此Gitelson和 Merzlyak（1994)提出绿色归一化植被指数 (Green $N D V I$ ) 有效地避免了 $675 \mathrm{~nm}$ 附近Chl光谱吸 收的影响, 提高了 NDVI对高 Chl含量叶片的预测精 度。Holben等(1983)研究了植被光谱与Chl浓度的关 
系, 研究表明叶片光谱的“红边” $\left(\lambda_{\text {re }}\right)$ 能够指示叶片 Chl的状态, 尽管不同类型植物的Chl含量与 $\lambda_{\mathrm{re}}$ 的定 量关系存在一些差异, 但 $\lambda_{\mathrm{re}}$ 与这些叶片的 Chl含量 之间均呈现高度正相关。Chappelle等(1992)和 Peñuelas等(1995a)在研究中提出用窄波段的反射光 谱指数来确定叶片绝对和相对的Chl浓度。然而这些 色素和叶片反射光谱关系的研究大多只在一个或几 个近缘种上测试过, 缺少不同叶片发育阶段和跨物 种的普适性研究。Sims和Gamon (2002)通过对多个 不同树种和功能型的幼叶、成熟叶和老叶的研究发 现, 在Chl总量降到 $0.04 \mathrm{mmol} \cdot \mathrm{m}^{-2}$ 之前, $445 \mathrm{~nm}$ 处的 光谱反射率 $\left(R_{445}\right)$ 保持稳定, 且 Chl在此处的光吸收 最小, 进而提出了用 $R_{445}$ 消除叶片结构对光谱反射 的影响, 建立了改良红边比值指数 $\left(m S R_{705}\right)$ 和改良 红边归一化植被指数 $\left(m N D_{705}\right)$, 减小了叶片表面反 射差异引起的误差, 广泛用于不同植被类型 $\mathrm{Chl}$ 含 量的估算。许改平等(2014)测定了不同温度下盆栽 毛竹(Phyllostachys edulis)实生苗的色素含量和反射 光谱, 结果发现色素含量与光谱反射率及微分光谱 在绿光区和红光区有显著或极显著的相关关系, 说 明反射光谱特征及其参数可用来估算叶片色素含量, 进而监测毛竹高温受伤害程度。在时间尺度上, Sampson等(2003)通过分析小型机载光谱成像仪 (CASI) 获得的光谱数据, 得出 $R_{750}$ 和 $R_{710}$ 的比值关系 可以用来监测森林Chl密度的季节性变化。此外, 在 估算Chl a 和Chl b含量的研究中, 色素简单比值指 数 $(P S S R)$ 、色素归一化指数 $(P S N D)$ 、反射光谱比值 分析(RARS)等参数的应用比较广泛(Chappelle et al., 1992; Blackburn, 1998)。

\section{2 类胡萝卜素}

类胡萝卜素(Car)在植物光合作用中不仅吸收 传递光能, 还具有保护Chl的功能。一方面, 当照射 植物的光照强度超过植物光合系统的利用能力时, 叶黄素(属于Car)循环(xanthophyll cycle)会将过剩的 光能散失掉, 以避免光合系统遭受光破坏(DemmigAdams \& Adams, 1996)。Gamon等(1992)在叶片尺度 上证明植物在 $531 \mathrm{~nm}$ 处的光谱反射率可以敏感的 反映叶黄素循环组分间的相互转换, 进而建立了生 理反射指数 $(P R I)$ 。Gamon和Surfus (1999)的研究表 明, 植物在暗适应下的 $P R I$ 与充分光适应下的 $P R I$ 之 差可以很好地反映叶黄素循环库的大小。当植物叶 片由暗处转到光下后, $P R I$ 的变化可以很好地反映
紫黄质(A)和玉米黄质(Z)的快速转变过程。彭涛等 (2009)的研究表明: 无论是在单叶水平还是冠层水 平测定的光谱反射特征, 均与叶黄素循环之间存在 极显著的线性相关关系; 冠层水平的光谱反射特征 能很好地反映植物群体的叶黄素循环的脱环化状 态。不同种植物对过剩光能的耗散机制并不完全相 同。角质、蜡质层较厚或有较多表皮毛的植物叶片 对光谱的反射情况也不尽相同, 对于这些植物叶黄 素循环的光谱响应关系还需要进行更多更深入的研 究。另一方面, 当叶片衰老或受到外界环境的胁迫 时, Car的存在可以延缓Chl的快速分解, 维持光合 作用的进行 (Biswal, 1995; Merzlyak \& Gitelson, 1995)。Gamon等(1992)提出的生理反射指数 $(P R I)$ 可 以用来估测Car/Chl。Peñuelas等(1995a)建立了结构 不敏感色素指数 (SIPI), 消除了叶片结构的影响, 可 以测定不同条件下不同植物叶片中Car和Chl a 的相 对变化; Merzlyak等(1999)研究发现色素组成变化 引起的叶片衰老和果实成熟会影响670和500 nm处 光谱反射率的差值, 进而建立了对Car/Chl敏感的植 物衰老反射指数 $(P S R I)$ 。Sims和Gamon (2002)在研 究中指出 $P R I$ 不受空间尺度、叶片结构和物种的限 制, 与Car/Chl密切相关。Gitelson等(2002)在对不同 色素含量和色素组成的不同树种叶片的反射光谱研 究中发现 $510 \mathrm{~nm}$ 附近的光谱反射率的倒数对Car的 含量最敏感, 但Chl也会影响这些光谱波段, 为了消 除Chl对510 nm附近光谱反射率的影响, 选用 550 和 $700 \mathrm{~nm}$ 建立了类胡萝卜素反射指数 $\left(C R I_{550}\right.$ 和 $\left.C R I_{700}\right)$ 来评估Car的含量。随后, Gitelson等(2006)又提出改 良的类胡萝卜素反射指数 $(m C R I)$, 提高了对Car含 量的预测精度。王福民等(2009)分析了所得波段范 围内所有归一化光谱反射指数与水稻(Oryza sativa) 叶片Car含量之间的关系, 发现 $N D_{(1536,707)}$ 可以较好 地估算Car含量; 杨杰等(2010)的研究提出光谱反射 指数 $S R_{(723,770)}$ 和归一化光谱反射指数 $N D_{(770,713)}$ 对水 稻叶片Car含量的预测具有较好的准确性和稳定性。

\section{3 植物营养元素对反射光谱的影响}

植物体内的氮(N)元素是氨基酸、蛋白质的组成 元素之一, 也是Chl、核酸、多种辅酶、维生素、植 物激素的主要成分(de Vries et al., 1974; de Vries, 1975), 植物营养元素亏缺导致的各种缺素症状都 会引起反射光谱的变化。 $\mathrm{N}$ 元素可作为评价植被光 
合作用强度和植被营养状况的一个重要指标(王渊, 2008)。N素亏缺引起的叶片颜色、厚度以及形态结 构的变化可以通过植物的反射光谱的变化反映出来 (Yoder \& Pettigrew-Crosby, 1995)。磷(P)元素和钾(K) 元素与植物生理活动也有十分密切的联系, $\mathrm{P}$ 元素 直接参与光合作用的同化和光合磷酸化; 而 $\mathrm{K}$ 元素 在光合作用中则主要担负气孔的调节、活化与光合 作用有关的酶、参与同化物的运输等重要的生理功 能。水分作为营养元素 $N 、 P 、 K$ 的运输者, 直接影 响 $\mathrm{N} 、 \mathrm{P} 、 \mathrm{~K}$ 的运输效率及其在植物体不同位置的含 量, 进而影响植物的生理活动。

在叶片尺度上, Stone等(1996)的研究表明植株 $\mathrm{N}$ 光谱指数 $(P N S I)$ 与小麦叶片的 $\mathrm{N}$ 吸收量显著相关, 且不受生长发育时期的影响, 用PNSI来指导施肥能 显著改善氮素利用效率, 减少因过度施肥对环境造 成的污染。Tarpley等(1999)研究表明红边波段(700 或716 nm)和短波近红外波段(755-920 nm和1 000 $\mathrm{nm})$ 的比值为作物叶层氮浓度估算提供了良好的精 度和准度。李雪飞等(2011)对紫叶稠李(Prunus virginiana) 的研究中发现, 紫叶稠李叶片的光谱反射 率在可见光波段(720-760 nm) 和近红外区(760-800 $\mathrm{nm})$ 与叶片 $\mathrm{N}$ 含量呈极显著的正相关。在冠层尺度上, Everitt等(1987)的研究表明植物叶片在500-750 nm 的光谱反射率与植物叶片含 $\mathrm{N}$ 量的相关性很高, 并 由此提出用550-600 $\mathrm{nm}$ 与 800-900 nm反射率的比 值来检测植株的N素营养状况。Lee等(2007)证实了 用735 $\mathrm{nm}$ 冠层光谱反射率的一阶导数 $\left(d R / d \lambda_{735}\right)$ 来 估测水稻叶层 $\mathrm{N}$ 含量的可行性。Zhu等(2008)在研究 中发现比值植被指数 $R V I_{(870,660)}$ 和 $R V I_{(810,660)}$ 与水稻 和小麦的叶片 $\mathrm{N}$ 积累量 $(L N A)$ 的相关性最好。Stroppiana等(2009)在对水稻叶片冠层光谱的研究中发现 可见光波段 $483 \mathrm{~nm}$ 和 $503 \mathrm{~nm}$ 的归一化组合 $N D_{(483,503)}$ 可以较好地估算植物 $\mathrm{N}$ 浓度 $(P N C)$ 。刘根华等(2011) 测定了不同施 $\mathrm{N}$ 水平的二年生山核桃(Carya $c a$ thayensis)实生苗的 $\mathrm{N}$ 素含量和反射光谱, 发现随着 $\mathrm{N}$ 素浓度的增加, 山核桃在可见光区的反射率逐渐 减小, 红边位置向长波方向移动, Green $N D V I$ 等多 个光谱指数可以表征山核桃的 $\mathrm{N}$ 素水平; 而 $P R I$ 随 $\mathrm{N}$ 素浓度先上升后下降, 在 $1.5 \mathrm{mmol} \cdot \mathrm{L}^{-1} \mathrm{~N}$ 素浓度下达 到最大值, 表明在该浓度下叶黄素循环活力最强, 最能有效地保护光合机构。在更大的空间尺度上, 张霞等(2003)利用机载的实用型模块化成像光谱仪
(OMIS)在北京小汤山地区获取航空高光谱遥感图 像, 运用红边、光谱吸收特征分析方法和逐步回归 算法, 进行了从高光谱遥感图像直接获取小麦 $\mathrm{N}$ 含 量的方法探索和可行性研究。然而, 冠层反射光谱 易受到传感器姿态、大气吸收、植被冠层结构、土 壤背景等因素的综合影响, 所建立的 $\mathrm{N}$ 素营养监测 模型精度还有待进一步提高(田永超等, 2010)。

与 $\mathrm{N}$ 素相比, $\mathrm{P}$ 和 $\mathrm{K}$ 对植物光谱特征的影响不明 显, 相关的研究也较少。Al-Abbas等(1974)发现近红 外波段存在有K元素的敏感波段; Milton等(1991)的 研究发现缺 $\mathrm{P}$ 叶片与正常叶片光谱存在一定的差异; Ayala-Silva和Beyl (2005)在研究中发现412-770 nm 是 $\mathrm{P}$ 和 $\mathrm{K}$ 元素的共同敏感波段; 但这些研究都没有 定量地给出反射光谱与 $\mathrm{P} 、 \mathrm{~K}$ 元素的关系。易时来等 (2010)研究了锦橙(Citrus sinensis 'Jincheng')叶片的 光谱反射与叶片中 $\mathrm{K}$ 含量的关系, 建立了锦橙叶片 K含量的回归模型。余天霞等(2014)研究指出微分技 术可以提高叶片P含量与叶片光谱信息的相关性。 朱文静(2014)采用相关分析法篮选基于偏振度特征 与番茄叶片参考值含量有显著关系的敏感波长，提 取出 $N 、 P 、 K$ 共有的敏感波长为655.408、744.482和 $850.578 \mathrm{~nm}$, 而 $\mathrm{N} 、 \mathrm{P} 、 \mathrm{~K}$ 特有的敏感波长依次分别为 380.487、914.562和556.664 nm, 并以敏感波长处的 偏振度为特征建立了MLR、PCR、PLS模型。总之, P 和 $K$ 引起的植物体生理变化在反射光谱上的响应尚 未研究清楚, 因此要阐明植物体内 $\mathrm{P}$ 和 $\mathrm{K}$ 含量与反 射光谱特征的关系, 还需要进行更深入的研究。

\section{4 植物光合与叶绿素荧光的变化及其光谱 响应规律}

当植物接收到超过本身光合作用所需的能量时 就要耗散掉过剩的光能，以避免“光氧化”或“光抑 制”。一种方式是天线色素(685 nm) 和反应中心Chl a 分子(695 nm) 在光系统II (PSII) 以荧光的形式向外 发出, 即光化学猝灭 $\left(q_{\mathrm{P}}\right)$; 另一种方式主要是以热 耗散的形式向外发出, 即非光化学猝灭 $(N P Q)$, 这 会引起相应的反射光谱发生变化。反射光谱与植物 光合生理活动关系的研究方面, 目前国际上主要有 生理反射指数 $(P R I)$ 和苂光比值指数 (FRI) (Dobrowski et al., 2005)两个重要指标。

生理反射指数 $(P R I)$ 是描述植物生理变化的一 个重要光谱指数, $P R I$ 是在研究叶黄素循环时首次 
提出来的(Gamon et al., 1992), 之后 $P R I$ 被证明与 $\mathrm{Car}$ 和Chl之间的比率的相关性也非常高, 利用 $P R I$ 的这一特性也可以定量估测植物的生理变化。不同 的是, 由于叶黄素循环的变化时间尺度是瞬间的, 适合描述短时间内植物的生理性胁迫; Car/Chl发生 变化的周期相对长一些, 适合用以描述长期发生的 胁迫, 但在特定时刻, 这两种因素对 $P R I$ 的影响究 竟哪种方式占有主导地位尚未可知(Sims \& Gamon, 2002)。另外有研究发现 $P R I$ 为利用植物反射光谱估 算光合效率提供了一个新方法, 可以用来反映植物 的光能利用效率 $(L U E)$ 。在时间尺度上, Peñuelas等 (1994)的研究表明 $P R I$ 可以用来探测 $L U E$ 的日变化; Stylinski等(2002)的研究表明 $N D V I$ 与 $\mathrm{CO}_{2}$ 净吸收量 的关系不显著, 而 $P R I$ 相比叶片 $\mathrm{Chl}$ 含量能更好地反 映常绿灌木的 $L U E$ 的季节变化。而在空间尺度上, 研究表明在叶片尺度(Peñuelas et al., 1995b)、冠层尺 度(Peñuelas et al., 1997a)和景观尺度上(Nichol et al., 2000; Peñuelas \& Inoue, 2000)都获得了 PRI和 $L U E 之$ 间较为稳定的关系; 吴朝阳和牛铮(2008)研究表明 随着空间尺度的变化, 水分、 $\mathrm{N}$ 素含量、叶面积指数 和太阳高度角等因素会对 $P R I$ 和 $L U E$ 的相关性产生 干扰，从而削弱 $P R I$ 和 $L U E$ 的关系。Peñuelas等(2004) 对木犀科一种灌木的研究表明 $P R I$ 与叶片的净光合 速率 $\left(P_{\mathrm{n}}\right)$ 呈线性相关。此外, 在不同树种的叶片和冠 层尺度, 以及在短时间和季节性尺度上, $P R I$ 多次被 证明与 PSII的实际光量子产量 $\left(\Delta F / F_{\mathrm{m}}\right)$ 和 $N P Q$ 具有 很高的相关性(Evain et al., 2004; Guo \& Trotter, 2004; Rascher et al., 2007; Peguero-Pina et al., 2008; Panigada et al., 2009; Osório et al., 2012)。

稳态叶绿素荧光 $\left(F_{\mathrm{s}}\right)$ 是指植物在无饱和闪光的 恒定光照条件下发射出的苂光, 它与植物光合作用 的状态有着密切的联系(Dobrowski et al., 2005)。有 学者发现可以利用 $F_{\mathrm{s}}$ 的变化实时监测植物的光合生 理变化和水分变化(Zarco-Tejada et al., 2000a, 2000b, 2003a; Dobrowski et al., 2005), 这开辟了监测植被 生理变化的新途径。但是, $F_{\mathrm{s}}$ 对表观光谱反射率的贡 献率很低(小于 $2 \%$, Zarco-Tejada et al., 2003a), 尽管 如此, 有科学家发现, 在温度和光照的双重和重度 胁迫试验条件下, FRI还是能够比较好地跟踪胁迫 前后 $\mathrm{CO}_{2}$ 同化速率, 气孔阻力等的降低以及胁迫之 后的逐渐恢复过程(Dobrowski et al., 2005), 但水分 胁迫条件下 $F R I$ 的变化尚未得到证实, 需要进一步
研究。

\section{5 植物反射光谱与碳氮水耦合关系的综合 分析及研究展望}

生态系统碳-氮-水循环是驱动生态系统变化的 关键过程。生态系统碳-氮-水循环在不同尺度存在 着一定的耦合平衡关系, 但目前我们对碳-氮-水循 环耦合的生物控制机制尚未了解清楚。高光谱遥感 作为一种能实时、非损伤性地获得植物生理生态信 息的手段, 在研究生态系统碳-氮-水循环的耦合机 制过程中具有独特的优势。通过高光谱遥感获得的 植物反射光谱能够及时地反映植物的水分状况、色 素含量、养分状况和光合荧光等生理指标及其内在 的相互关联(图1), 可以为揭示植物对生态系统碳氮-水循环的作用机制和实现以提高水、碳、氮效率 为目标的生物调控提供技术和方法支持; 这对于估 测全球气候变化背景下生态系统的碳收支状况和植 被生产力具有重要的意义。

当前, 对反射光谱与植物生理生态指标相关性 的研究已经有很多的报道, 但探讨由水分状况变化 引起的植物生理变化与反射光谱关系的研究还相对 较少。水分作为引起植物反射光谱发生变化的双重 因素(结构性因素和生理性因素), 既是植物叶片的 重要组成部分, 在NIR和SWIR反射区具有特定的光 谱吸收区间; 又诱导植物叶片发生生理变化, 引起 反射光谱在VIS区的变化, 且各类生理变化对反射 光谱的影响具有一定的叠加交互效应。现有的研究 在甄别植物水分状况变化和由水分变化引起的各类 生理变化的作用机制, 量化其各自对反射光谱的影 响程度方面还有所欠缺, 尚不足以解释植物体水分 状况变化造成的复杂的光谱响应机制, 因此, 未来 有关应用反射光谱探测植物水分及生理活动的研究 应主要集中在以下方面。

植物水分状况的直接光谱响应: 通过不同植物 敏感光谱波段的提取和组合, 建立光谱指数与植物 水分含量的相关性; 修正现有的水分估算模型的参 数, 提高现有模型的精度; 探索新的普适性好、精 度高的水分敏感光谱指数和模型。

植物水分胁迫造成的其他生理指标变化的光谱 响应机制探索: 通过比较和分析水分胁迫和非水分 胁迫条件下植物色素库及比率、养分状况和光合苂 光指标的光谱响应差异, 甄选和提取水分胁迫下的 


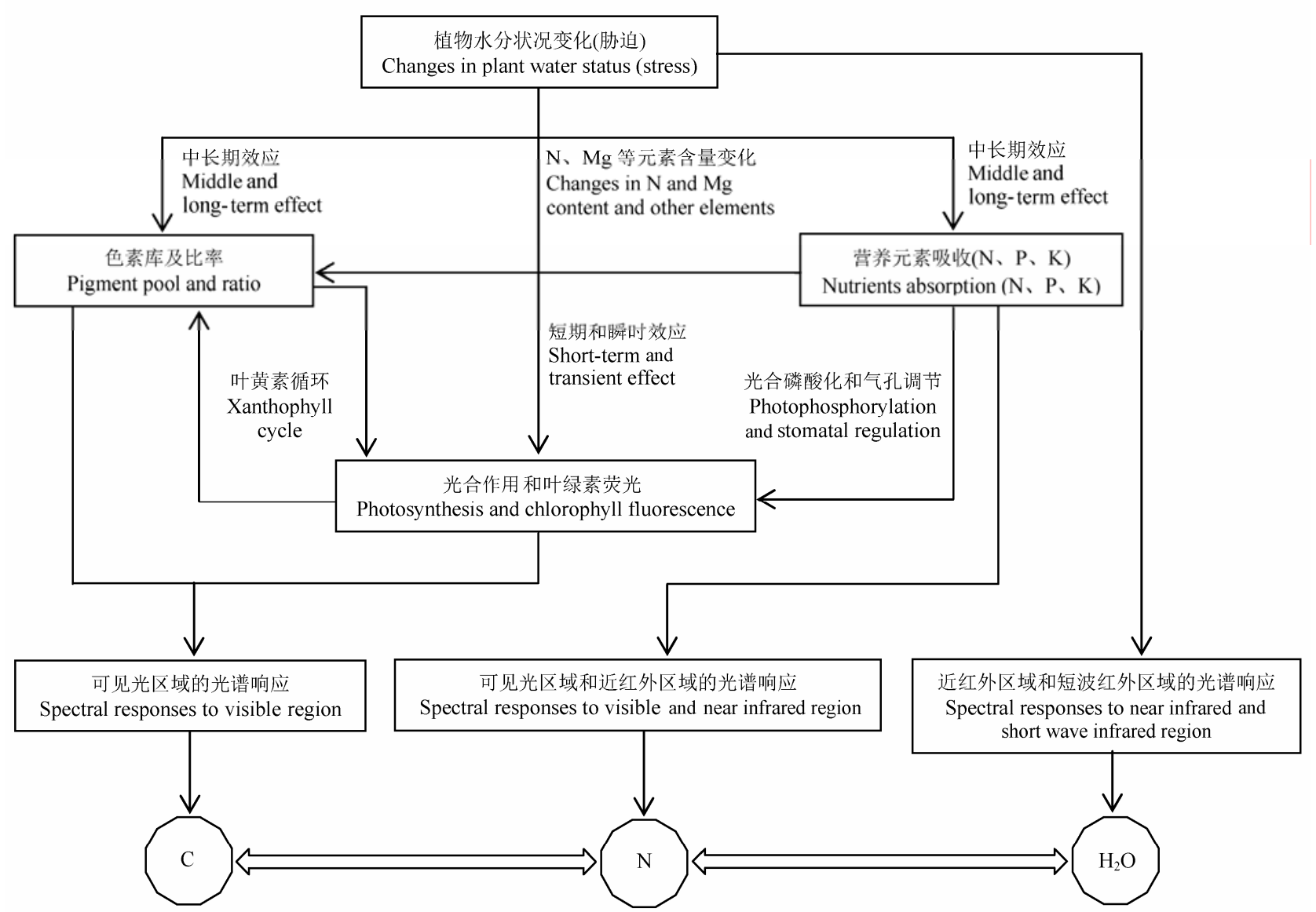

图1 植物反射光谱对碳-氮-水耦合关系的响应机制。

Fig. 1 The response mechanism of plant spectral reflectance to carbon-nitrogen-water coupling relationship.

色素敏感光谱指数、养分敏感光谱指数和光合荧光 生理敏感光谱指数, 进而利用这些敏感光谱指数更 加全面和准确地监测植物的水分及生理状况。

基金项目 国家自然科学基金(31290223)。

\section{参考文献}

Al-Abbas AH, Barr R, Hall JD, Crane FL, Baumgardner MF (1974). Spectra of normal and nutrient-deficient maize leaves. Agronomy Journal, 66, 16-20.

Ayala-SilvaT, Beyl CA (2005). Changes in spectral reflectance of wheat leaves in response to specific macronutrient deficiency. Advances in Space Research, 35, 305-317.

Baret F, Jacquemoud S, Guyot G, Leprieur C (1992). Modeled analysis of the biophysical nature of spectral shifts and comparison with information content of broad bands. Remote Sensing of Environment, 41, 133-142.

Biswal B (1995). Carotenoid catabolism during leaf senescence and its control by light. Journal of Photochemistry and Photobiology B: Biology, 30, 3-13.

Blackburn GA (1998). Quantifying chlorophylls and caroten- iods at leaf and canopy scales: An evaluation of some hyperspectral approaches. Remote Sensing of Environment, 66, 273-285.

Bowman WD (1989). The relationship between leaf water status, gas exchange, and spectral reflectance in cotton leaves. Remote Sensing of Environment, 30, 249-255.

Carter GA (1993). Responses of leaf spectral reflectance to plant stress. American Journal of Botany, 80, 239-243.

Ceccato P, Flasse S, Tarantola S, Jacquemoud S, Grégoire JM (2001). Detecting vegetation leaf water content using reflectance in the optical domain. Remote Sensing of Environment, 77, 22-33.

Chappelle EW, Kim MS, McMurtrey III JE (1992). Ratio analysis of reflectance spectra (RARS): An algorithm for the remote estimation of the concentrations of chlorophyll A, chlorophyll B, and carotenoids in soybean leaves. Remote Sensing of Environment, 39, 239-247.

Danson FM, Steven MD, Malthus TJ, Clark JA (1992). High-spectral resolution data for determining leaf water content. International Journal of Remote Sensing, 13, 461-470.

de Vries FWTP (1975). The cost of maintenance processes in 
plant cells. Annals of Botany, 39, 77-92.

de Vries FWTP, Brunsting AHM, Van Laar HH (1974). Products, requirements and efficiency of biosynthesis a quantitative approach. Journal of theoretical Biology, 45, 339377.

Demmig-Adams B, Adams III WW (1996). The role of xanthophyll cycle carotenoids in the protection of photosynthesis. Trends in Plant Science,1, 21-26.

Dobrowski SZ, Pushnik JC, Zarco-Tejada PJ, Ustin SL (2005). Simple reflectance indices track heat and water stress-induced changes in steady-state chlorophyll fluorescence at the canopy scale. Remote Sensing of Environment, 97, 403-414.

Evain S, Flexas J, Moya I (2004). A new instrument for passive remote sensing: 2. Measurement of leaf and canopy reflectance changes at $531 \mathrm{~nm}$ and their relationship with photosynthesis and chlorophyll fluorescence. Remote Sensing of Environment, 91, 175-185.

Everitt JH, PettitRD, Alaniz MA (1987). Remote sensing of broom snakeweed (Gutierrezia sarothrae) and spiny aster (Aster spinosus). Weed Science, 35, 295-302.

Gamon JA, Peñuelas J, Field CB (1992). A narrow-waveband spectral index that tracks diurnal changes in photosynthetic efficiency. Remote Sensing of Environment, 41, 35-44.

Gamon JA, Rahman AF, Dungan JL, Schildhauer M, Huemmrich KF (2006). Spectral Network (SpecNet) - What is it and why do we need it? Remote Sensing of Environment, 103, 227-235.

Gamon JA, Surfus JS (1999). Assessing leaf pigment content and activity with a reflectometer. New Phytologist, 143, 105-117.

Gao BC (1996). NDWI-A normalized difference water index for remote sensing of vegetation liquid water from space. Remote Sensing of Environment, 58, 257-266.

Gao BC, Goetz AFH (1995). Retrieval of equivalent water thickness and information related to biochemical components of vegetation canopies from AVIRIS data. Remote Sensing of Environment, 52, 155-162.

Gitelson A, Merzlyak MN (1994). Spectral reflectance changes associated with autumn senescence of Aesculus hippocastanum L. and Acer platanoides L. leaves spectral features and relation to chlorophyll estimation. Journal of Plant Physiology, 143, 286-292.

Gitelson AA, Keydan GP, Merzlyak MN (2006). Three-band model for noninvasive estimation of chlorophyll, carotenoids, and anthocyanin contents in higher plant leaves. Geophysical Research Letters, 33, doi: 10.1029/2006GL 026457.

Gitelson AA, Zur Y, Chivkunova OB, Merzlyak MN (2002). Assessing carotenoid content in plant leaves with reflectance spectroscopy. Photochemistry and Photobiology, 75, 272-281.

Grace J, Nichol C, Disney M, Lewis P, Quaife T, Bowyer P
(2007). Can we measure terrestrial photosynthesis from space directly, using spectral reflectance and fluorescence? Global Change Biology, 13, 1484-1497.

Graeff S, Claupein W (2007). Identification and discrimination of water stress in wheat leaves (Triticum aestivum L.) by means of reflectance measurements. Irrigation Science, 26, 61-70.

Guo JM, Trotter CM (2004). Estimating photosynthetic light-use efficiency using the photochemical reflectance index: Variations among species. Functional Plant Biology, 31, 255-265.

Holben BN, Schutt JB, McMurtrey III J (1983). Leaf water stress detection utilizing thematic mapper bands 3, 4 and 5 in soybean plants. International Journal of Remote Sensing, 4, 289-297.

Hunt Jr ER, Rock BN (1989). Detection of changes in leaf water content using near- and middle-infrared reflectances. Remote Sensing of Environment, 30, 43-54.

Imanishi J, Sugimoto K, Morimoto Y (2004). Detecting drought status and LAI of two Quercus species canopies using derivative spectra. Computers and Electronics in Agriculture, 43, 109-129.

Inoue Y, Morinaga S, Shibayama M (1993). Non-destructive estimation of water status of intact crop leaves based on spectral reflectance measurements. Japanese Journal of Crop Science, 62, 462-469.

Jacquemoud S, Biswal F (1990). PROSPECT: A model of leaf optical properties spectra. Remote Sensing of Environment, 34, 75-91.

Kramer PJ, Boyer JS (1995). Water Relations of Plants and Soils. Academic Press, San Diego, USA.

Lee YJ, Yang CM, Chang KW, Shen Y (2007). A simple spectral index using reflectance of $735 \mathrm{~nm}$ to assess nitrogen status of rice canopy. Agronomy Journal, 100, 205-212.

Li H, Lascano RJ, Barnes EM, Booker J, Wilson LT, Bronson KF, Segarra E (2000). Multispectral reflectance of cotton related to plant growth, soil water and texture, and site elevation. Agronomy Journal, 93, 1327-1337.

Li XF, Han TT, Dong Y, Wu M, Shen X (2011). Relationships between spectral reflectance and pigment or nitrogen concentrations in leaves of Prunus virginiana 'Schubert'. Scientia Silvae Sinicae, 47(8), 75-81. (in Chinese with English abstract) [李雪飞, 韩甜甜, 董彦, 吴曼, 沈向 (2011). 紫叶稠李叶片色素及氮含量与其光谱反射特性 的相关性. 林业科学, 47(8), 75-81.]

Liu GH, Huang JQ, Pan CX, Wang ZJ, Zheng BS, Jin SH (2011). Analysis of the nitrogen levels in leaves of Hickory seedling by reflectance spectra. Scientia Silvae Sinicae, 47, 165-171. (in Chinese with English abstract) [刘根 华, 黄坚钦, 潘春霞, 王正加, 郑炳松, 金松恒 (2011). 基于反射光谱的山核桃幼苗氮素营养状况分析. 林业 科学, 47, 165-171.]

www.plant-ecology.com 
Merzlyak MN, Gitelson A (1995). Why and what for the leaves are yellow in autumn? On the interpretation of optical spectra of senescing leaves (Acer platanoides L.). Journal of Plant Physiology, 145, 315-320.

Merzlyak MN, Gitelson AA, Chivkunova OB, Rakitin VY (1999). Non-destructive optical detection of pigment changes during leaf senescence and fruit ripening. Physiologia Plantarum, 106, 135-141.

Milton NM, Eiswerth BA, Ager CM (1991). Effect of phosphorus deficiency on spectral reflectance and morphology of soybean plants. Remote Sensing of Environment, 36, 121-127.

Minolta K (1989). Chlorophyll meter SPAD-502. In: Radiometric Instruments Operations ed. Instruction Manual. Minolta, Osaka, Japan.

Muttiah RS (2002). From Laboratory Spectroscopy to Remotely Sensed Spectra of Terrestrial Ecosystems. Springer, Dordrecht, Netherlands.

Nichol CJ, Huemmrich KF, Black TA, Jarvis PG, Walthall CL, GraceJ, Hall FG (2000). Remote sensing of photosynthetic-light-use efficiency of boreal forest. Agricultural and Forest Meteorology, 101, 131-142.

Osório J, Osório ML, Romano A (2012). Reflectance indices as nondestructive indicators of the physiological status of Ceratonia siliqua seedlings under varying moisture and temperature regimes. Functional Plant Biology, 39, 588-597.

Pan PF, Yang WN, Dai XA (2013). Vegetation moisture content model based on principal component analysis. Remote Sensing for Land \& Resources, 25(3), 38-42. (in Chinese with English abstract) [潘佩芬, 杨武年, 戴晓爱 (2013). 基于主成分分析的植被含水率模型. 国土资源遥感, 25(3), 38-42.]

Panigada C, Rossini M, Meroni M, Marzuoli R, Gerosa G, Colombo R (2009). Indicators of ozone effects on Fagus sylvatica $\mathrm{L}$. by means of spectroradiometric measurements. Rivista Italiana di Telerilevamento, 41, 3-20.

Peguero-Pina JJ, Morales F, Flexas J, Gil-Pelegrín E, Moya I (2008). Photochemistry, remotely sensed physiological reflectance index and de-epoxidation state of the xanthophyll cycle in Quercus coccifera under intense drought. Oecologia, 156, 1-11.

Peng T, Yao G, Gao HY, Li PM, Wang WW, Sun S, Zhao SJ (2009). Relationship between xanthophyll cycle and photochemical reflectance index measured at leaf or canopy level in two field-grown plant species. Acta Ecologica Sinica, 29, 1987-1993. (in Chinese with English abstract) [彭涛, 姚广, 高辉远, 李鹏民, 王未未, 孙山, 赵世杰 (2009). 植物叶片和冠层光化学反射指数与叶黄素循环 的关系. 生态学报, 29, 1987-1993.]

Peñuelas J, Baret F, Filella I (1995a). Semi-empirical indices to assess carotenoids/chlorophyll a ratio from leaf spectral reflectance. Photosynthetica, 31, 221-230.
Peñuelas J, Filella I, Gamon JA (1995b). Assessment of photosynthetic radiation-use efficiency with spectral reflectance. New Phytologist, 131, 291-296.

Peñuelas J, Gamon JA, Fredeen AL, Merino J, Field CB (1994). Reflectance indices associated with physiological changes in nitrogen- and water-limited sunflower leaves. Remote Sensing of Environment, 48, 135-146.

Peñuelas J, Inoue Y (1999). Reflectance indices indicative of changes in water and pigment contents of peanut and wheat leaves. Photosynthetica, 36, 355-360.

Peñuelas J, Inoue Y (2000). Reflectance assessment of canopy $\mathrm{CO}_{2}$ uptake. International Journal of Remote Sensing, 21, 3353-3356.

Peñuelas J, Llusia J, Pinol J, Filella I (1997a). Photochemical reflectance index and leaf photosynthetic radiation-useefficiency assessment in Mediterranean trees. International Journal of Remote Sensing, 18, 2863-2868.

Peñuelas J, Munné-Bosch S, Llusià J, Filella I (2004). Leaf reflectance and photo-and antioxidant protection in field-grown summer-stressed Phillyrea angustifolia optical signals of oxidative stress? New Phytologist, 162, 115-124.

Peñuelas J, Pinol J, Ogaya R, Filella I (1997b). Estimation of plant water concentration by the reflectance Water Index WI (R900/R970). International Journal of Remote Sensing, 18, 2869-2875.

Rascher U, Nichol CJ, Small C, Hendricks L (2007). Monitoring spatio-temporal dynamics of photosynthesis with a portable hyperspectral imaging system. Photogrammetric Engineering \& Remote Sensing, 73, 45-56.

Rollin EM, Milton EJ (1998). Processing of high spectral resolution reflectance data for the retrieval of canopy water content information. Remote Sensing of Environment, 65, 86-92.

Rouse JJ, Haas RH, Schell JA, Deering DW (1974). Monitoring Vegetation Systems in the Great Plains with Erts. NASA Special Publication, Washington, USA. 309.

Sampson PH, Zarco-Tejada PJ, Mohammed GH, Miller JR, Noland TL (2003). Hyperspectral remote sensing of forest condition: Estimating chlorophyll content in tolerant hardwoods. Forest Science, 49, 381-391.

Sandholt I, Rasmussen K, Andersen J (2002). A simple interpretation of the surface temperature/vegetation index space for assessment of surface moisture status. Remote Sensing of environment, 79, 213-224.

Sellers PJ, Dickinson RE, Randall DA, Betts AK, Hall FG, Berry JA, Collatz GJ, Denning AS, Mooney HA, Nobre CA, Sato N, Field CB, Henderson-Sellers A (1997). Modeling the exchanges of energy, water, and carbon between continents and the atmosphere. Science, 275, 502-509.

Shen Y, Niu Z, Wang W, Xu YM (2005). Establishment of leaf water content models based on derivative spectrum variables. Geography and Geo-Information Science, 21(4), 
16-19. (in Chinese with English abstract) [沈艳, 牛铮, 王 汶, 徐永明 (2005). 基于导数光谱变量叶片含水量模型 的建立. 地理与地理信息科学, 21(4), 16-19.]

Sims DA, Gamon JA (2002). Relationships between leaf pigment content and spectral reflectance across a wide range of species, leaf structures and developmental stages. Remote Sensing of Environment, 81, 337-354.

Sims DA, Gamon JA (2003). Estimation of vegetation water content and photosynthetic tissue area from spectral reflectance: A comparison of indices based on liquid water and chlorophyll absorption features. Remote Sensing of Environment, 84, 526-537.

Stone ML, Solie JB, Raun WR, Whitney RW, Taylor SL, Ringer JD (1996). Use of spectral radiance for correcting in-season fertilizer nitrogen deficiencies in winter wheat. Transactions of the ASAE, 39, 1623-1631.

Stroppiana D, Boschetti M, Brivio PA, Bocchi S (2009). Plant nitrogen concentration in paddy rice from field canopy hyperspectral radiometry. Field Crops Research, 111, 119-129.

Stylinski C, Gamon J, Oechel W (2002). Seasonal patterns of reflectance indices, carotenoid pigments and photosynthesis of evergreen chaparral species. Oecologia, 131, 366-374.

Szilagyi J (2000). Can a vegetation index derived from remote sensing be indicative of areal transpiration? Ecological Modelling, 127, 65-79.

Tarpley L, Reddy KR, Sassenrath-Cole GF (1999). Reflectance indices with precision and accuracy in predicting cotton leaf nitrogen concentration. Crop Science, 40, 1814-1819.

Tian YC, Yang J, Yao X, Cao WX, Zhu Y (2010). Monitoring canopy leaf nitrogen concentration based on leaf hyperspectral indices in rice. Acta Agronomica Sinica, 36, 1529-1537. (in Chinese with English abstract) [田永超, 杨杰, 姚霞, 曹卫星, 朱艳 (2010). 利用叶片高光谱指 数预测水稻群体叶层全氮含量. 作物学报, 36, 15291537.]

Tian YC, Zhu Y, Cao WX, Dai TB (2004). Relationship between canopy reflectance and plant water status of wheat. Chinese Journal of Applied Ecology, 15, 2072-2076. (in Chinese with English abstract) [田永超, 朱艳, 曹卫星, 戴廷波 (2004). 小麦冠层反射光谱与植株水分状况的 关系. 应用生态学报, 15, 2072-2076.]

Tyree MT, Jarvis PG (1982). Water in tissues and cells. In: Lange OL, Nobel PS, Osmond CB, Ziegler H eds. Physiological Plant Ecology II. Springer, Berlin. 35-77.

Ustin SL, Roberts DA, Pinzón J, Jacquemoud S, Gardner M, Scheer G, Castañeda CM, Palacios-Orueta A (1998). Estimating canopy water content of Chaparral shrubs using optical methods. Remote Sensing of Environment, 65, 280-291.

Verhoef W (1984). Light scattering by leaf layers with applica- tion to canopy reflectance modeling: The SAIL model. Remote Sensing of Environment, 16, 125-141.

Veroustraete F, Sabbe F, Eerens H (2002). Estimation of carbon mass fluxes over Europe using the C-Fix model and Euroflux data. Remote Sensing of Environment, 83, 376-399.

Wang FM, Huang JF, Wang XZ (2009). Normalized difference ratio pigment index for estimating chlorophyll and cartenoid contents of in leaves of rice. Spectroscopy and Spectral Analysis, 29, 1064-1068. (in Chinese with English abstract) [王福民, 黄敬峰, 王秀珍 (2009). 水稻叶片叶绿 素、类胡萝卜素含量估算的归一化色素指数研究. 光谱 学与光谱分析, 29, 1064-1068.]

Wang Y (2008). Information Extraction of Rape Nitrogen Concentration Using Remotely Sensed Data at Different Levels. PhD dissertation, Zhejiang University, Hangzhou. (in Chinese) [王渊 (2008). 不同水平油菜氮素含量遥感 信息提取方法研究. 博士学位论文, 浙江大学, 杭州.]

Wang ZH, Liu JD, Liu L, Wu DR, Qiu MJ, Feng YD (2013). Regional agro-drought simulation based on remote sensing technology. Bulletin of Soil and Water Conservation, 33(5), 96-100, 122. (in Chinese with English abstract) [王 治海, 刘建栋, 刘玲, 鸟定荣, 邱美娟, 俸玉端 (2013). 基于遥感信息的区域农业干旱模拟技术研究. 水土保 持通报, 33(5), 96-100, 122.]

Wu ZY, Niu Z (2008). Review of retrieval light use efficiency using photochemical reflectance index (PRI). Journal of Plant Ecology (Chinese Version), 32, 734-740. (in Chinese with English abstract) [吴朝阳, 牛铮 (2008). 光化 学植被指数估算植物光能利用率的研究进展. 植物生 态学报, 32, 734-740.]

Xu GP, Wu XB, Liu F, Wang YK, Gao Y, Zuo ZJ, Wen GS, Zhang RM (2014). The correlation between the pigment content and reflectance spectrum in Phyllostachys edulis leaves subjected to high temperature. Scientia Silvae Sinicae, 50(5), 41-48. (in Chinese with English abstract) [许 改平, 吴兴波, 刘芳, 王玉魁, 高岩, 左照江, 温国胜, 张汝民 (2014). 高温胁迫下毛竹叶片色素含量与反射 光谱的相关性. 林业科学, 50(5), 41-48.]

Xu HY, Jia JH, Liu LY, Peng DL, Li CJ, Wu YP (2015). Drought monitoring in Huang-Huai-Hai Plain using the multi-drought indices. Remote Sensing Technology and Application, 2015, 30, 25-32. [徐焕预, 贾建华, 刘良云, 彭代亮, 李存军, 吴耀平 (2015). 基于多源干旱指数的 黄淮海平原干旱监测. 遥感技术与应用, 30, 25-32.]

Yang J, Tian YC, Yao X, Cao WX, Zhu Y (2010). Estimating leaf carotenoid content with hyperspectral parameters in rice. Chinese Journal of Plant Ecology, 34, 845-854. (in Chinese with English abstract) [杨杰, 田永超, 姚霞, 曹 卫星, 朱艳 (2010). 利用高光谱参数反演水稻叶片类胡 萝卜素含量. 植物生态学报, 34, 845-854.]

www.plant-ecology.com 
Yi SL, Deng L, He SL, Zheng YQ, Mao SS (2010). A spectrum based models for monitoring leaf potassium content of Citrus sinensis (L) cv. Jincheng orange. Scientia Agricultura Sinica, 43, 780-786. (in Chinese with English abstract) [易时来, 邓烈, 何绍兰, 郑永强, 毛莎莎 (2010). 锦橙叶片钾含量光谱监测模型研究. 中国农业科学, 43, 780-786.]

Yoder BJ, Pettigrew-Crosby RE (1995). Predicting nitrogen and chlorophyll content and concentrations from reflectance spectra (400-2500 nm) at leaf and canopy scales. Remote Sensing of Environment, 53, 199-211.

Yu GR, Miwa T, Nakayama K, Matsuoka N, Kon H (2000). A proposal for universal formulas for estimating leaf water status of herbaceous and woody plants based on spectral reflectance properties. Plant and Soil, 227, 47-58.

Yu TX, Luo HX, Zou YQ, Wang J (2014). On correlation between leaf spectral reflectance and agricultural parameter of Beibei 447 Jincheng orange leaves. Journal of Southwest China Normal University (Natural Science Edition), 39(5), 33-37. (in Chinese with English abstract) [余天霞, 罗红霞, 邹扬庆, 王俊 (2014). 北碚447锦橙叶片农学 参数与其反射光谱的相关性研究. 西南师范大学学报 (自然科学版), 39(5), 33-37.]

Zarco-Tejada PJ, Miller JR, Mohammed GH, Noland TL (2000a). Chlorophyll fluorescence effects on vegetation apparent reflectance: I. Leaf-level measurements and model simulation. Remote Sensing of Environment, 74, 582-595.

Zarco-Tejada PJ, Miller JR, Mohammed GH, Noland TL, Sampson PH (2000b). Chlorophyll fluorescence effects on vegetation apparent reflectance: II. Laboratory and airborne canopy-level measurements with hyperspectral data. Remote Sensing of Environment, 74, 596-608.

Zarco-Tejada PJ, Pushnik JC, Dobrowski S, Ustin SL (2003a). Steady-state chlorophyll a fluorescence detection from canopy derivative reflectance and double-peak red-edge effects. Remote Sensing of Environment, 84, 283-294.

Zarco-Tejada PJ, Rueda CA, Ustin SL (2003b). Water content estimation in vegetation with MODIS reflectance data and model inversion methods. Remote Sensing of Environment, 85, 109-124.

Zhang J, Wu JJ, Zhou L, Lei TJ, Liu M (2012). Comparative study on remotely sensed methods of monitoring agricultural drought based on MODIS data. Remote Sensing Information, 27(5), 48-54. (in Chinese with English abstract) [张洁, 武建军, 周否, 雷添杰, 刘明 (2012). 基 于MODIS数据的农业干旱监测方法对比分析. 遥感信 息, 27(5), 48-54.]

Zhang X, Liu LY, Zhao CJ, Zhang B (2003). Estimating wheat nitrogen concentration with high spectral resolution image. Journal of Remote Sensing, 7, 176-181. (in Chinese with English abstract) [张霞, 刘良云, 赵春江, 张兵 (2003). 利用高光谱遥感图像估算小麦氮含量. 遥感学报, 7, 176-181.]

Zhu WJ (2014). Study on Determination of Nitrogen, Phosphorus, Potassium Content and Interactive Effect in Greenhouse Tomato Leaves Using Polarized SpectraHyperspectral Multidimensional Optical Information. $\mathrm{PhD}$ dissertation, Jiangsu University, Zhenjiang. (in Chinese) [朱文静 (2014). 基于偏振-高光谱多维光信息的番茄氮 磷钾及交互作用检测研究. 博士学位论文, 江苏大学, 镇江.]

Zhu Y, Yao X, Tian YC, Liu XJ, Cao WX (2008). Analysis of common canopy vegetation indices for indicating leaf nitrogen accumulations in wheat and rice. International Journal of Applied Earth Observation and Geoinformation, 10, 1-10.

责任编委: 李 彦 责任编辑: 李 敏

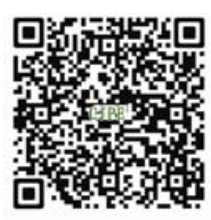

植物生态学报官网

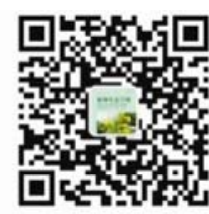

微信订阅号

期刊及学科

相关信息发布

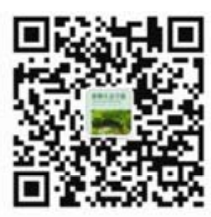

微信服务号

稿件状态查询 全文检索测览 